\title{
DEVELOPMENT \& EVALUATION OF A SURGICAL STEREO VISION LASER-SCANNING SURFACE RECOGNITION SYSTEM
}

Pascal Wettmann ${ }^{1}$, Florian Coigny ${ }^{1}$, Gregor Imboden ${ }^{2}$, Bruno Knobel ${ }^{2}$, Erik Schkommodau ${ }^{1}$

\author{
${ }^{1}$ Institute for Medical and Analytical Technologies, University of Applied Sciences North Western Switzerland \\ ${ }^{2}$ Naviswiss AG, Laufen, Switzerland \\ pascal.wettmann@fhnw.ch
}

\begin{abstract}
A stereo vision surface scanning system using a laser with a mounted diffractive element was developed and evaluated. The system was tested on accuracy, performance and usability in a surgical environment. The system can be used complementary to an optical, mobile tracking system using the same stereo camera.

Results show a satisfying accuracy and viability for surgical applications. It is possible to match and register surface data to segmented CT data with satisfying accuracy.
\end{abstract}

Keywords: 3D-Scanner, Stereo Vision, Surgery, Registration, Laser Diffraction, Tracking

\section{Introduction}

The aim was to develop and evaluate a 3D scanning system, which: can be easily combined with a mobile tracking system, works in a surgical environment and can be used on close distance. In a previous project a mobile, optical tracking system for surgical requirements was developed, working with a monochrome stereo camera and a passive black and white marker. Adding a laser and projecting a patter on the scenery, the 3D point-cloud of an objects surface can be obtained. Consequently, with almost no extra hardware a tracking system can be used as $3 \mathrm{D}$ scanner.

A 3D scanning system combined with a tracking system already exists in a larger format. The tracking system is a static tower (around 2 meters high), which tracks the mobile 3D scan unit [5]. The innovation is the combination of optical tracking and 3D scanning using the same stereo camera and image stream.

Having the surface, a registration with preoperative data is possible [4], which can be used for navigation purposes.

\section{Methods}

To build up the stereo camera system, two "Baumer Optronic" monochrome cameras (Type: TXF20) equipped with Computar lenses with focal length 12 millimetres were used. Cameras are synchronized using a hardware trigger. A red $10 \mathrm{~mW}$ laser with a $7 \times 7$ point matrix diffraction head from Edmund Optics was used (fig. 1).

In $270 \mathrm{~mm}$ distance both images are congruent aside from perspective deviations. The working area is around 165 $\mathrm{mm}$ times $115 \mathrm{~mm}$.

Exposure time has to be adjusted, that a marker with a black and white pattern on it is still clearly visible, in order to be able to realize marker recognition with the same image stream.
Laser point (blob) detection in the 2D image is done by applying a global threshold [3].

Point mapping from left to right camera image is determined using epipolar geometry [1]. 3D reconstruction is performed using forward intersection [2].

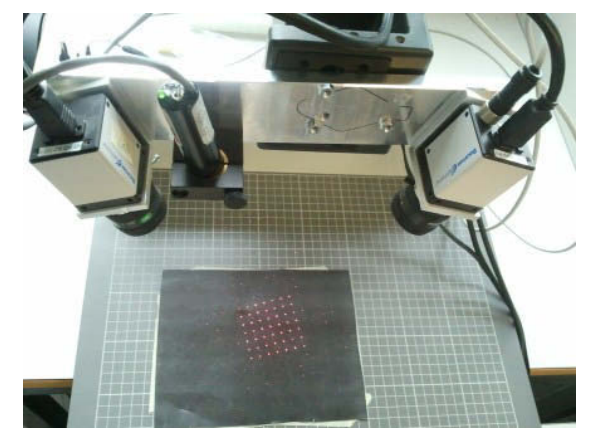

Figure 1: Setup of the stereo vision system with a diffractive laser pattern.
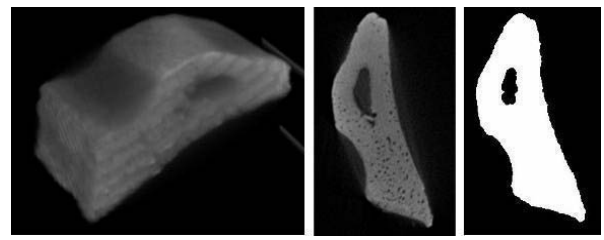

Figure 2: Bone part scanned in the micro-CT, segmented and finally transformed into an STL file using marching cubes algorithm.

In order to get satisfying results, outlier handling is necessary. Three countermeasures were used. First of all, the minimal distance of the forward intersection was checked. Secondly, the nearest distance to any other recorded point in the same frame was evaluated and in the third place the nearest distance to a point in the previous frame is considered. For all of these criteria a threshold value was determined experimentally, comparing the results to a reference scanner. As reference a GOM Atos I scanner was used.

For analyses commercial software "GOM Inspect" was used. Recorded point cloud was compared to STL files generated from the reference 3D scanner and the segmented micro-CT (fig. 2).

\section{Results}

Accuracy of the system is satisfying and sufficient to match preoperative data. Bone tissue can be scanned (fig. 4 and 5). Flesh and fluid drops strongly absorb the laser, consequently scanning flesh, fluids and bone simultane- 
ously using a static threshold as described above is problematic (fig. 4). Adjusting the cameras exposure time, tissue to be scanned can be affected. Using the proposed outlier handling, quality of the scanned points can be improved dramatically. Performance is around 50 points per second, which is rather low compared to other systems.

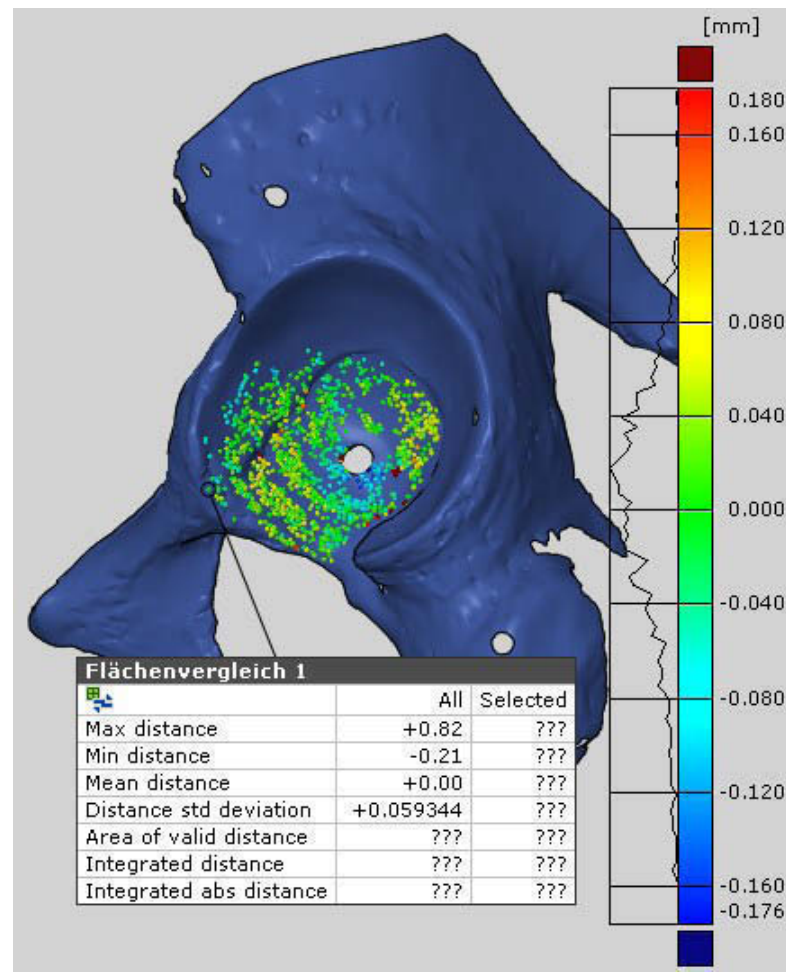

Figure 3: Result of scanning an artificial acetabulum for 30 seconds compared to the GOM Atos I scanner. Conditions: lighting on surface: 10’000 lux.
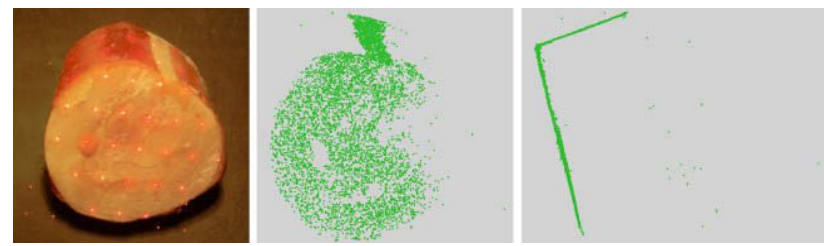

Figure 4: Result of scanning a wet bone with some flesh on it. Conditions as in figure 3.

\section{Discussion}

The system exhibits good properties, which enable the scanner to be used in a surgical environment. It is promising, that the system can deal with reflections, since this is one of the most important issues in surgery. The accuracy is high enough to use it as reference for previous planned surgeries. Commercial spot 3D scanners exhibit a similar accuracy [6]. Stripe 3D scanners achieve less accurate results [6]. The probably largest drawback is the rather low scanning speed. Whilst common spot 3D scanners are in the range of 100 points per second [6], this system only reaches around 50. Stripe 3D scanners reach even $1000+$ points per second [6]. Performance was not in the focus in this project, so it definitely can be increased.

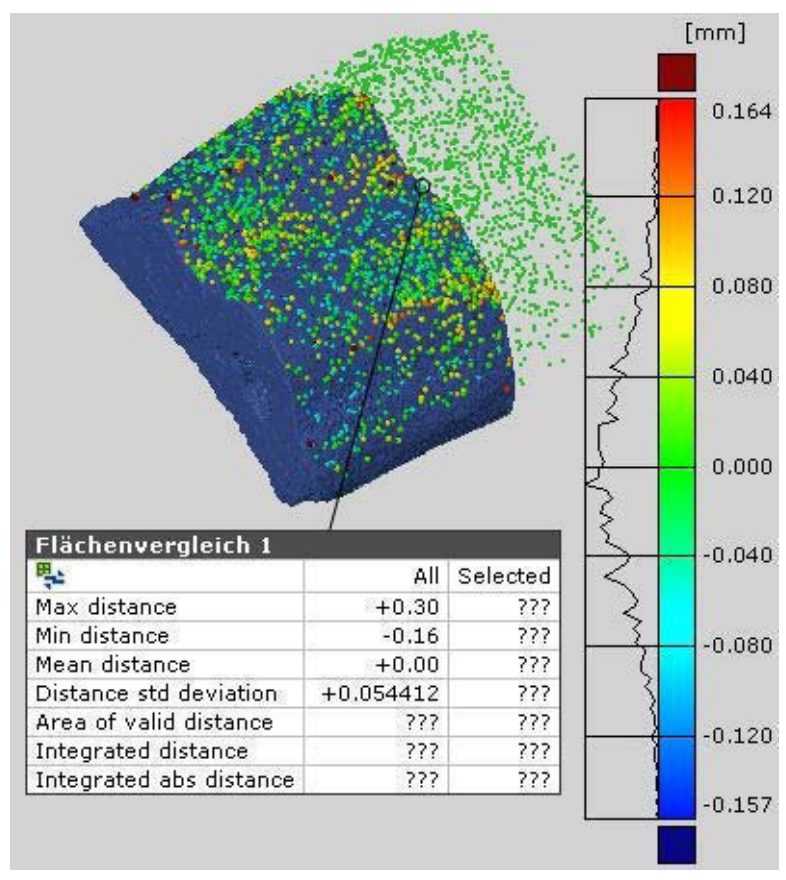

Figure 5: Result of scanning a bone with dry surface for two minutes compared to a segmented micro-CT. Conditions as in figure 3. Blue model from micro-CT is smaller, since the bone didn't fit into the workspace, hence just a part is considered.

Proposed outlier handling has to be adjusted properly. More restrictive thresholds improve the result, however, decrease the performance and the system's ability to scan gaps and leaps.

The system has a great potential in computer assisted surgery.

\section{Bibliography}

[1] Richard Hartley, Andrew Zisserman; Multiple View Geometry in Computer Vision; Chapter 9: Epipolar Geometry and the Fundamental Matrix; Cambridge; 2003.

[2] Wolfgang Förstner, Bernhard Weobel, Didel Paderes, Robert Craig, Clive Fraser, John Dolloff; Manual of Photogrammetry fifth edition; Chapter 11: Analytical Photogrammetric Operations; ASPRS; 2004.

[3] Wilhelm Burger, Mark J. Burge; Digital Image Processin; Springer; 2008.

[4] D. Troitzsch1, J. Hoffmann1, F. Dammann2, D. Bartz3, S. Reinert1; Registration using three-dimensional laser surface scanning for navigation in oral and craniomaxillofacial surgery; Thieme; 2003.

[5] Steinbichler, T-Track CS \& T-Scan CS; URL: http://www.steinbichler.de/produkte/surfacescanning/3d-digitalisierung/t-trackcs.html\#\%C3\%BCbersicht [mar. 2013]

[6] Nunzio Alberto Borghese, Giancarlo Ferrigno, Guido Baroni, Antonio Pedotti, Stefano Ferrari, Riccardo Savarè , Phoenix Tools ; Autoscan: A Flexible and Portable 3D Scanner, IEEE; 1998.

[7] Chris Boehnen, Patrick Flynn; Accuracy of 3D Scanning Technologies in a Face Scanning Scenario; IEEE; 2005. 